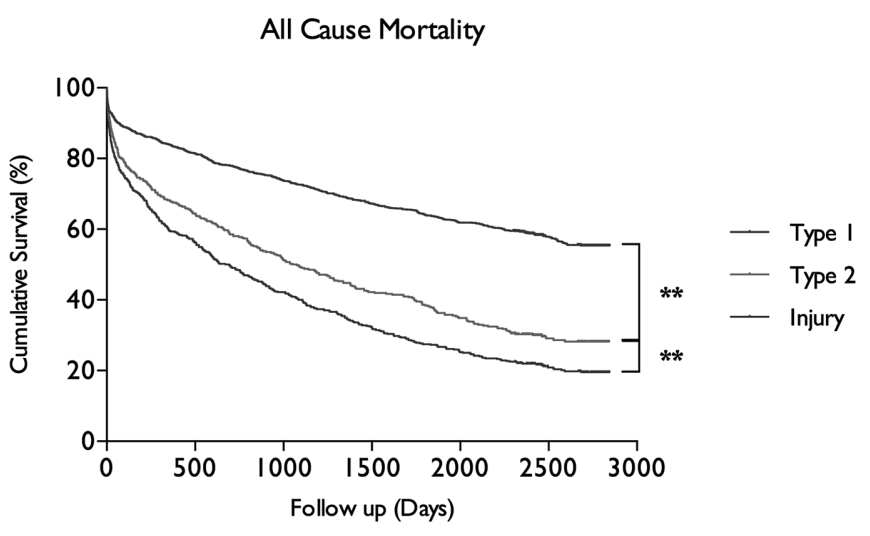

Abstract 113 Figure 1 Kaplan Meier curve displaying time to death stratified by classification of myocardial infarction or myocardial injury. Log-rank test for comparison. ${ }^{* *}$ denotes $p<0.01$

\section{PERSISTENCE OF HAEMOGLOBIN DEGRADATION PRODUCTS WITHIN INFARCT SCAR TISSUE AFTER ST- ELEVATION MYOCARDIAL INFARCTION: INCIDENCE, CORRELATES AND IMPLICATIONS FOR LEFT VENTRICULAR REMODELLING}

\begin{abstract}
${ }^{1} J a c l y n$ Carberry*, 'David Carrick, ${ }^{2}$ Caroline Haig, 'Sam Rauhalammi, 'Nadeem Ahmed, ${ }^{1}$ Ify Mordi, ${ }^{1}$ Margaret McEntegart, ${ }^{1}$ Mark Petrie, ${ }^{1}$ Hany Eteiba, ${ }^{1}$ Stuart Hood, ${ }^{1}$ Stuart Watkins, ${ }^{1}$ Mitchell Lindsay, ${ }^{1}$ Andrew Davie, ${ }^{1}$ Ahmed Mahrous, ${ }^{2}$ lan Ford, ${ }^{1}$ Naveed Sattar, ${ }^{1}$ Paul Welsh, ${ }^{1}$ Keith G Oldroyd, ${ }^{1}$ Aleksandra Radjenovic, ${ }^{1}$ Colin Berry. ${ }^{1}$ BHF Glasgow Cardiovascular Research Centre, Institute of Cardiovascular and Medical Sciences, University of Glasgow; ${ }^{2}$ Robertson Centre for Biostatistics, University of Glasgow; *Presenting Author
\end{abstract}

\subsection{6/heartjnl-2016-309890.114}

Background Myocardial haemorrhage is a prognostically important complication of acute ST-elevation myocardial infarction (STEMI). Persistence of haemoglobin degradation products within infarct scar tissue and the potential clinical significance have not been investigated.

Methods and Results Patients who sustained an acute STEMI were enrolled in a cohort study (BHF MR-MI; ClinicalTrials. gov: NCT02072850). 211 patients (mean (SD) age 57 (11) years, $77 \%$ male) had evaluable $\mathrm{T} 2 *$ cardiac magnetic resonance (CMR) imaging (1.5 Tesla) 2 days 6 months post-MI. Myocardial haemorrhage was defined as a hypointense infarct core with T2* signal <20ms. At 2 days, 79 (37\%) patients had evidence of myocardial haemorrhage. At 6 months, 47 $(59 \%)$ patients had a hypointense infarct core and $32(41 \%)$ did not. None of the patients had de novo haemorrhage after the first CMR scan. Clinical associates of a persistent hypointense core at 6 months included hypertension (odds ratio (95\% confidence interval) $0.28(0.08,0.95) ; \mathrm{p}=0.040)$, heart rate $(1.08(1.03,1.13) ; \mathrm{p}=0.001)$, systolic blood pressure $(1.06(1.01,1.12) ; \mathrm{p}=0.015)$, neutrophil count $(2.19$ $(1.01,4.74) ; \mathrm{p}=0.048)$, left anterior descending as culprit artery $(9.26(1.26,67.99) ; \mathrm{p}=0.029)$, infarct size $(1.10$ $(1.03,1.17) ; \mathrm{p}=0.004)$ and initial haemorrhage size $(1.34$ $(1.06,1.66) ; \mathrm{p}=0.012)$. A hypointense infarct core with T2*-mapping at 6 months was associated with worsening LV end-diastolic volume (regression coefficient (95\% confidence interval) $15.43(1.35,29.50) ; \mathrm{p}=0.032)$ and worsening left ventricular ejection fraction $(-4.15(-7.40,-0.89) ; \mathrm{p}=0.013)$. Conclusion Persistence of haemoglobin degradation products at 6 months post-STEMI is common and prognostically important.

\section{PERSISTENCE OF INFARCT ZONE OEDEMA AT 6 MONTHS AFTER ACUTE ST-ELEVATION MYOCARDIAL INFARCTION: INCIDENCE, PATHOPHYSIOLOGY AND ASSOCIATION WITH LEFT VENTRICULAR REMODELLING}

1Jaclyn Carberry*, 'David Carrick, ${ }^{2}$ Caroline Haig, 'Sam Rauhalammi, ${ }^{1}$ Nadeem Ahmed, ${ }^{1}$ Ify Mordi, ${ }^{1}$ Margaret B McEntegart, ${ }^{1}$ Mark Petrie, ${ }^{1}$ Hany Eteiba, ${ }^{1}$ Stuart Hood, ${ }^{1}$ Stuart Watkins, ${ }^{1}$ Mitchell Lindsay, ${ }^{1}$ Andrew Davie, ${ }^{1}$ Ahmed Mahrous, ${ }^{2}$ lan Ford, ${ }^{1}$ Naveed Sattar, ${ }^{1}$ Paul Welsh, ${ }^{1}$ Keith G Oldroyd, ${ }^{1}$ Aleksandra Radjenovic, ${ }^{1}$ Colin Berry. ${ }^{1}$ BHF Glasgow Cardiovascular Research Centre, Institute of Cardiovascular and Medical Sciences, University of Glasgow; ${ }^{2}$ Robertson Centre for Biostatistics, University of Glasgow; *Presenting Author

\subsection{6/heartjnl-2016-309890.115}

Background The natural history of persistent myocardial oedema after ST-elevation myocardial infarction (STEMI) is uncertain.

Methods and ResultsPatients who sustained an acute STEMI were enrolled in a cohort study (BHF MR-MI; ClinicalTrials. gov: NCT02072850). Cardiac magnetic resonance (CMR) imaging with T2-mapping of myocardial oedema was performed at 1.5 Tesla 2 days and 6 months post-MI. Myocardial oedema was defined as infarct signal intensity (S. I.) $>2$ standard deviations from the mean S. I. within a remote reference region. 283 STEMI patients (mean (SD) age 59 (12) years, $75 \%$ male) were enrolled. Infarct size was $18(13) \%$ of left ventricular (LV) mass. At 2 days, infarct zone T2 (ms) was higher than remote zone T2 $(62.9$ (5.2) vs. 49.7 (2.1); p $<0.001$ ), and this relationship persisted at 6 months (56.2 (4.1) vs. 49.7 (2.3); p < 0.001). Mean remote zone T2 did not change over time $(\mathrm{p}=0.840)$ whereas infarct zone T2 decreased $(\mathrm{p}<0.001)$. At 6 months, infarct zone oedema persisted in 177 (63\%) patients who were more likely to have a history of hypertension (regression coefficient $(95 \%$ confidence interval) $1.77(0.99,3.13)$; 0.053$)$ and a larger initial size of infarction $(1.02(1.00,1.05) ; \mathrm{p}=0.020)$ but less likely to have a history of previous PCI $(0.28$ (0.08, 0.99); $\mathrm{p}=$ 0.049 ), compared to patients without persistent oedema. In a multivariate analysis, infarct zone oedema at 6 months was associated with an increase in LV end-diastolic volume $(9.68$ (3.76, 15.61); $\mathrm{p}=0.001)$.

Conclusion Persistence of oedema within the infarct zone affected the majority of patients at 6 months post-STEMI, and was associated with the initial severity of STEMI and adverse LV remodelling.

\section{Imaging}

\section{PHYSIOLOGIST-DIRECTED STRESS MYOCARDIAL PERFUSION SCINTIGRAPHY PROTOCOL IS SAFE: A SINGLE SITE PRE- AND POST-INTERVENTION STUDY}

${ }^{1}$ Sean Zheng* ${ }^{2}$ Sergei Pavlitchouk, ${ }^{2}$ Andrew Kelion, ${ }^{2}$ Nikant Sabharwal. ${ }^{1}$ King's College Hospital; ${ }^{2}$ John Radcliffe Hospital; *Presenting Author

\subsection{6/heartjnl-2016-309890.116}

Introduction Vasodilator stress as part of MPS has existed for decades. There is wide national variation in procedural protocols with many centres advocating physician presence during stress MPS. The suggested safety profile of the adenosine-2A agonist Regadenoson lends itself to physiologist-led stress MPS without the need for physician presence. We investigated the 
safety of stress MPS with a physiologist-led protocol using Regadenoson as the primary vasodilator agent, compared with an earlier physician-led protocol.

Methods Our centre prefers dynamic stress and will combine it with vasodilator stress as required. Earlier local audit data suggested Regadenoson was safer than other pharmacological stressors in keeping with published data. This resulted in the introduction of physiologist-led stress MPS in September 2011 and replacement of the previous physician-led protocol. Data were prospectively stored on a database. We reviewed all documented serious adverse events (AE) (VF/VT, cardiac arrest, unplanned admission, death) associated with dynamic and vasodilator stress MPS between 1 September 2008 and 30 August 2014. Outcome data between the two periods were analysed: physician-led (September 2008 to August 2011) and physiologist-led (September 2011 to August 2014).

Results In all, 11297 patients underwent stress MPS (physician-led, 5321; physiologist-led, 5976). Compared to the physician-led period, there were fewer patients in sinus rhythm in the physiologist-led period $(86.4 \%$ vs $91.4 \%, \mathrm{P}<$ 0.01 ) and more patients deemed to have a high prognostic cardiovascular risk post-scan $(11.7 \%$ vs $9.2 \%, \mathrm{P}<0.01)$ (Table 1). Vasodilator stress was used more often in the physiologist-led period $(43.9 \%$ vs $27.9 \%, \mathrm{P}<0.01)$. Regadenoson was the main vasodilator in physiologist-led period (64.9\%); Dipyridamole in physician-led period (83.9\%) (Table 1). There were 8 cases of serious $\mathrm{AE}$ (4 in each period) (0.07\%): VF/ VT ( 1 and 3 cases in physician and physiologist-led periods respectively), cardiac arrest $(2,2)$, admission $(2,3)$ and one death $(1,0)$. Relative risk of serious $\mathrm{AE}$ was 0.89 (95\% CI: 0.2-3.6) for patients in the physiologist-led period, relative to those in the physician-led period (Table 2).

Conclusions This retrospective study compared physiologist-led stress MPS using regadenoson as the primary vasodilator agent with a previous physician-led protocol. Physiologist-led stress MPS was not associated with an increased rate of serious AE, with no deaths. Regadenoson stress MPS may be used safely in a physiologist-led setting without immediate physician presence.

Abstract 116 Table 1 Demographics and Stress mechanism

\begin{tabular}{llll}
\hline & Physician-led & Physiologist-led & P-Value \\
\hline Demographics & & & \\
$\mathrm{N}$ & 5321 & 5976 & \\
Male & $56.6 \%$ & $60.1 \%$ & $<0.01$ \\
Age (median) & 66 & 66 & $\mathrm{NS}$ \\
Sinus rhythm & $91.4 \%$ & $86.4 \%$ & $<0.01$ \\
Prognostic risk post-MPS high $¥$ & $9.2 \%$ & $11.7 \%$ & $<0.01$ \\
Prognostic risk post-MPS low $\%$ & $73.0 \%$ & $68.8 \%$ & $<0.01$ \\
Stress mechanism & & & $<0.01$ \\
Physical (treadmill/bicycle) & $72.1 \%$ & $56.1 \%$ & $<0.01$ \\
Pharmacological (all) & $27.9 \%$ & $43.9 \%$ & $<0.01$ \\
Dobutamine & $10.6 \%$ & $1.1 \%$ & $<0.01$ \\
Dipyridamole & $83.9 \%$ & $29.8 \%$ & $<0.01$ \\
Adenosine & $5.4 \%$ & $4.2 \%$ & $<0.01$ \\
Regadenoson & $0.2 \%$ & $64.9 \%$ & \\
\hline
\end{tabular}

$\ddagger$ prognostic risk calculated post-MPS - high: $>3 \%$ estimated annualised risk of major adverse cardiovascular event, low: $<1 \%$
Abstract 116 Table 2 Serious adverse events

\begin{tabular}{llll}
\hline & Physician-led & Physiologist-led & \\
\hline Total cases & $4(0.1 \%)$ & $4(0.1 \%)$ & $0.9(0.2-3.6) \dagger$ \\
VFNT & 1 & 3 & \\
Cardiac arrest & 2 & 2 & \\
Emergency admission & 2 & 3 & \\
Death & 1 & 0 & \\
\hline
\end{tabular}

$\dagger 95 \%$ confidence interval

\section{OPTIMISATION AND REPRODUCIBILITY OF 18F- FLUORIDE POSITRON EMISSION TOMOGRAPHY AND COMPUTED TOMOGRAPHY IN PATIENTS WITH AORTIC STENOSIS}

Tania Pawade*, Timothy Cartlidge, David Newby, Dweck Marc. University of Edinburgh; *Presenting Author

\subsection{6/heartjnl-2016-309890.117}

Background 18F-Fluoride positron emission tomography (PET) and computed tomography (CT) can measure disease activity and progression in aortic stenosis. We aimed to optimise imaging methodology, analysis and scan-rescan reproducibility.

Methods Fifteen patients with aortic stenosis underwent $18 \mathrm{~F}$ fluoride PET-CT twice within 1 month. We compared nongated PET and non-contrast CT, with a modified approach that incorporated contrast CT and ECG-gated PET. We explored a range of image analysis techniques including estimation of blood pool activity at differing vascular sites and a most-diseased segment (MDS) approach.

Results Contrast-enhanced ECG-gated PET-CT provided superior spatial localisation of $18 \mathrm{~F}$-fluoride uptake that permitted localisation to individual valve leaflets (Figure). Scan-rescan reproducibility was markedly improved using enhanced analysis techniques leading to a reduction in variability from $25 \%$ to $<10 \%$ (tissue-to-background MDS: mean value 1.55, difference 0.05 , limits of agreement $-0 \cdot 10$ to $0 \cdot 20$ ).

Conclusion Optimised 18F-fluoride PET-CT provides excellent spatial resolution and scan-rescan reproducibility. It holds major promise as a marker of disease activity in aortic stenosis and has major potential as a biomarker end-point of trials of novel therapies in aortic stenosis.

\section{SINGLE SOURCE, DUAL ENERGY CT FOR THE ASSESSMENT OF CALCIFIED CORONARY ARTERY DISEASE}

${ }^{1}$ Benjamin Clayton, ${ }^{2}$ Franchesca Wotton*, ${ }^{2}$ Carl Roobottom, ${ }^{2}$ Gareth Morgan-Hughes. ${ }^{1}$ Torbay and South Devon NHS Foundation Trust; ${ }^{2}$ Derriford Hospital; * Presenting Author

10.1136/heartjnl-2016-309890.118

Introduction Arterial calcification can limit the visualisation of vessel lumen at CT coronary angiography (CTCA). Dual energy CT (DECT) using two x-ray spectra of differing energy, either from distinct sources or using novel detectors and a single source, offers novel approaches to this problem. It allows the generation of images depicting objects as if they have been subjected to a specific photon energy $(\mathrm{keV})$ rather than a polychromatic beam (virtual monochromatic images). With improved material identification, based on its attenuation coefficient at each $\mathrm{keV}$ and transformation into a linear combination of the two basis materials (material decomposition), it 